\title{
A randomized controlled trial to investigate the influence of low dose radiotherapy on immune stimulatory effects in liver metastases of colorectal cancer
}

Christoph Reissfelder ${ }^{1}$, Carmen Timke ${ }^{2}$, Hubertus Schmitz-Winnenthal ${ }^{1}$, Nuh N Rahbari ${ }^{1}$, Moritz Koch ${ }^{1}$, Felix Klug ${ }^{3}$, Falk Roeder ${ }^{2}$, Lutz Edler², Jürgen Debus², Markus W Büchler ${ }^{1}$, Philipp Beckhove², Peter E Huber ${ }^{2}$ and Jürgen Weitz ${ }^{1 *}$

\begin{abstract}
Background: Insufficient migration and activation of tumor specific effector $T$ cells in the tumor is one of the main reasons for inadequate host anti-tumor immune response. External radiation seems to induce inflammation and activate the immune response. This phase I/II clinical trial aims to evaluate whether low dose single fraction radiotherapy can improve T cell associated antitumor immune response in patients with colorectal liver metastases.

Methods/Design: This is an investigator-initiated, prospective randomised, 4-armed, controlled Phase I/II trial. Patients undergoing elective hepatic resection due to colorectal cancer liver metastasis will be enrolled in the study. Patients will receive 0 Gy, 0.5 Gy, 2 Gy or 5 Gy radiation targeted to their liver metastasis. Radiation will be applied by external beam radiotherapy using a $6 \mathrm{MV}$ linear accelerator (Linac) with intensity modulated radiotherapy (IMRT) technique two days prior to surgical resection. All patients admitted to the Department of General-, Visceral-, and Transplantion Surgery, University of Heidelberg for elective hepatic resection are consecutively screened for eligibility into this trial, and written informed consent is obtained before inclusion. The primary objective is to assess the effect of active local external beam radiation dose on, tumor infiltrating $T$ cells as a surrogate parameter for antitumor activity. Secondary objectives include radiogenic treatment toxicity, postoperative morbidity and mortality, local tumor control and recurrence patterns, survival and quality of life. Furthermore, frequencies of systemic tumor reactive T cells in blood and bone marrow will be correlated with clinical outcome.
\end{abstract}

Discussion: This is a randomized controlled patient blinded trial to assess the safety and efficiency of low dose radiotherapy on metastasis infiltrating $T$ cells and thus potentially enhance the antitumor immune response.

Trial registration: ClinicalTrials.gov: NCT01191632

Keywords: colorectal liver metastasis, low dose radiation, tumor specific T cells

\section{Background}

Colorectal cancer (CRC) is the most frequent cancer in the western world [1]. Advanced tumor stage with metastasis is one of the main reasons for the high mortality rate. At the time of diagnosis, approximately $30 \%$

\footnotetext{
* Correspondence: juergen.weitz@med.uni-heidelberg.de

'Department of General, Visceral and Transplantation Surgery, University

Hospital Heidelberg, Germany

Full list of author information is available at the end of the article
}

of patients have distant metastases which predominantly occur in the liver. Surgical removal of the tumor still remains the only curative approach [2,3]. In recent years survival rates in colorectal cancer have been further improved because of multimodal therapy concepts, especially in UICC stage III and IV patients. In parallel to these modern multimodal therapy concepts, novel and promising concepts including immunotherapeutic
C Biomed Central

(c) 2011 Reissfelder et al; licensee BioMed Central Ltd. This is an Open Access article distributed under the terms of the Creative Commons Attribution License (http://creativecommons.org/licenses/by/2.0), which permits unrestricted use, distribution, and reproduction in any medium, provided the original work is properly cited. 
strategies are actively being investigated to further improve clinical outcome.

Most patients with CRC have high numbers of tumor specific $\mathrm{T}$ cells (cytotoxic $\mathrm{T}$ cells and $\mathrm{T}$ helper cells) in their blood and bone marrow [4,5]. The molecular identification of tumor-associated antigens (TAA) has led to the development of antigen-specific immunotherapy targeting these antigens. To the present these strategies have failed to show efficacy in clinical practice. Potential reasons for this lack of efficacy include the inefficiency of $\mathrm{T}$ cell activation in vivo, insufficient migration of cytotoxic $\mathrm{T}$ cells into the tumor, the choice of irrelevant antigens, induction or application of monovalent $\mathrm{T}$ cells or the treatment of patients in highly advanced tumor stages [6]. In particular inadequate $T$ cell infiltration, characterized by selective recruitment of immune suppressive regulatory $\mathrm{T}$ cells as well as insufficient immigration of cytotoxic $\mathrm{T}$ cells [7-9] appears to be a serious hurdle, partially explainable by poorly activated endothelium of the tumor vessels [10]. Furthermore, the cytotoxic and secretory activity of tumor specific $\mathrm{T}$ cells may be inhibited by the immunosuppressive environment of the tumor stroma after immigration into the tumor.

Ionizing radiation therapy (RT) is an effective local cancer therapy, which kills cancer and other cells within the tumor stroma, including endothelial cells and intratumoral lymphocytes [11]. RT typically induces primarily mitotic cell death but also leads to apoptosis [12]. Radiation has also complex effects on the tumor microenvironment and microvessels, which can facilitate the homing of both antigen presenting and effector $\mathrm{T}$ cells to the tumor $[10,13]$.

Tumor cells killed by RT may be a good source of antigens for Dendritic Cell (DC) uptake and presentation to T cells. DCs are attracted by the altered microenvironment and undergo maturation after internalizing the debris. These dendritic cells can then present tumor specific antigens to T-cells following the processing of debris [14]. The possibility of using RT to promote tumor antigen-presentation by DC has been explored. Sublethal doses of ionising radiation have been described to enhance antitumor T-cell activity in such indirect ways in animal studies. Radiation has also been described to directly lead to an amplification of expression of MHC. This can render tumor cells more sensitive to the detection and lysis by specific T-cells [15]. Furthermore ionizing radiation can lead to activated endothelium thus facilitating the immigration of T-cells into the tumor. Overall, the available preclinical in vivo data support the hypothesis that RT may provide at least some of the necessary maturation signals.

So far, no systematic clinical analysis has been performed to investigate the immune stimulatory effects of low dose radiotherapy in patients with CRC. One issue to be addressed is the effective radiation dose to act as a form of immunotherapy. In fact, scant data are available with regard to the specific radiosensitivity of the different cellular components of the immune system. Studies describing the effects of RT on circulating immune cells are also lacking along with data on radiation effects on the microenvironment.

We here present the protocol of a clinical phase I/II trial with the aim to determine the active local external beam radiation dose resulting in tumor infiltrating $\mathrm{T}$ cells in patients with liver metastasis from CRC. The liver metastases will be irradiated two days prior to surgery using external beam radiotherapy. As primary endpoints we will determine the active local radiation dose leading to metastasis infiltrating $\mathrm{T}$ cells as a surrogate parameter for antitumor activity. Secondary objectives include local tumor control, survival, treatment toxicity as well as quality of life. Furthermore, blood cell transcriptomics and plasma-proteomics will be correlated with the amount of tumor reactive $\mathrm{T}$ cells in blood and bone marrow.

\section{Methods/Design}

\section{Trial population and patient recruitment}

This trial focuses on patients with resectable liver metastases of CRC. Patients scheduled for elective hepatic resection due to colorectal liver metastasis will be enrolled in this study. Only patients over 50 years of age are allowed to participate in this study due to the radiation protection law. Based on the preoperative imaging the surgeon determines if the liver metastasis can be resected in curative intent. Patients with unresectable liver metastasis, secondary malignancies, liver cirrhosis or previous radiotherapy to the upper abdomen will be excluded from the trial (see Table 1).

Patients who meet the eligibility criteria and provide written informed consent are randomly allocated to either study intervention to balance treatment groups

\section{Table 1 Eligibility criteria}

\begin{tabular}{l} 
Inclusion criteria \\
Liver metastasis of colorectal cancer \\
Metastasis is curatively resectable \\
Written informed consent of the patient \\
No evidence of active or former concurrent malignant diseases \\
\hline Exclusion criteria \\
Previous radiotherapy to the upper abdomen \\
Participation in other therapeutical trials \\
Liver cirrhosis \\
Pregnancy \\
No willingness on regular follow up care \\
Any extrahepatic disease
\end{tabular}


for all known and unknown potentially confounding factors. A block randomisation list is created via a computer system. Patients are randomised using consecutively numbered opaque envelopes prepared and sealed by an independent study nurse. The envelopes will be opened sequentially only after the participant's name is written on the appropriate envelope. Neither the screening investigators nor the investigators who inform the potential participants about this trial and enrolling the patients, have any knowledge of the block randomisation list or the previous allocations to treatment. There is no stratification for randomisation.

After informed consent, patients are randomized either to the control group - receiving no radiation - or the three irradiation treatment groups. Within the irradiation treatment groups patients will receive $0.5 \mathrm{~Gy}, 2$ Gy or 5 Gy single fraction radiation $48 \mathrm{~h}$ prior to resection. After the adjustment of an individual positioning device, computed tomography and, if needed, magnetic resonance imaging (MRI) treatment planning examinations are performed. After 3D inverse treatment planning and plan optimizing using VIRTUOS and KONRAD software, intensity modulated radiation therapy will be administered using five to nine $6 \mathrm{MV}$ linear acceleration (LINAC) photon beams. Immediately prior to the irradiation the setup error of the patient will be determined via In-Room-CT, and corrected if necessary.

The first blood sample will be obtained $(54 \mathrm{ml})$ before radiation, a second just prior to the start of the operation. A bilateral bone marrow sample (27 $\mathrm{ml}$ each) will be taken from both iliac crests after induction of general anaesthesia just before skin incision. Bone marrow sampling is optional for the patient. EDTA will be used as anticoagulant for bone marrow and blood samples. After hepatic resection a part of the tumor will be immediately removed and stored at $-80^{\circ} \mathrm{C}$ for further analyses.

On the postoperative day seven and at the follow-up/ restaging visits (4 weeks postoperatively, four and seven months postoperatively) additional venous blood samples $(54 \mathrm{ml})$ will be obtained. Quality of Life will be tested using the EORTC QLQ C-30 at four months and seven months postoperatively. Clinical examination will be performed and blood samples will be obtained by the investigators at all visits.

Curative surgery requires complete resection of all liver metastases, regardless of size, number, distribution, or width of resection margin. Liver operation will be carried out under low central venous pressure using a Péan clamp, ultrasonic dissector or vascular stapler. An intraoperative ultrasound of the liver will be performed in all patients to assess the relationship of the lesions to major vessels and to rule out additional lesions. The Brisbane 2000 terminology of hepatic anatomy and resections will be applied [16].

\section{Safety aspects and adverse events}

As the radiation dose is relatively low, very few side effects are expected. Potential adverse events (AE) include skin alterations like redness, temporary elevated transaminases, transient nausea or diarrhea. All possible AEs will be assessed by the RTOG Scale. These AEs rarely occur in conventional radiotherapy using considerably higher doses (neoadjuvant $5 \times 5$ Gy for rectal or gastric cancer, 54 Gy in pancreatic cancer) $[17,18]$.

\section{Study design}

This single fraction radiation on colorectal liver metastases trial is a registered (ClinicalTrials.gov NCT01191632), investigator-initiated, prospective, randomized controlled phase I/II trial to evaluate the optimal neoadjuvant, single fraction radiation dose for patients undergoing resection for colorectal liver metastases in curative intent. Patients will be randomised either to the control group -receiving no radiation- or the three treatment groups - receiving $0.5 \mathrm{~Gy}, 2 \mathrm{~Gy}$ or 5 Gy single fraction radiation to the metastasis $48 \mathrm{~h}$ prior to the operation. The treatment is offered to a heterogeneous group of patient of both sexes, covering a wide range of comorbidities. For the irradiation a highly conformal dose distribution to the tumor with $1 \mathrm{~cm}$ safety margin is delivered using a $6 \mathrm{MV}$ LINAC and intensity modulated radiotherapy (IMRT) treatment application. After inclusion of 20 patients an interims analysis will be performed. The final analysis will be performed within three month of the inclusion of the last patient. The trial design will not be changed without prior agreement of the ethics committee.

Translational studies will be carried out by the Department of Translational Immunology of $\mathrm{dkfz}$, the Radiation Oncology Departments of German Cancer Research Center (DKFZ) and the Surgery Departments of the University Hospital. This trial is an investigatorinitiated trial and is coordinated by the Department of General-, Visceral- and Transplantation Surgery and the Radiation Oncology Department of DKFZ. The Radiation Oncology Department of DKFZ is responsible for the overall trial management, trial registration, database management, quality assurance including monitoring and scientific program of all trial related meetings. The methods and the study design of this trial have the same structure as the previously published study protocol for patients with pancreatic cancer (BMC Cancer 2011; 11:34) [19].

\section{Ethical and legal considerations}

The final clinical trial protocol, the patient information and informed consent sheets were approved by the independent ethics committee of the University of Heidelberg S081/2008 (http://klinikum.uni-heidelberg.de). 
Furthermore, the study has been approved by the German Federal Authorities for Radiation Protection (Bundesamt fuer Strahlenschutz, Salzgitter, Trial Number Z5-22461/2-2009-002). Written informed consent is obtained from each patient in oral and written form before inclusion in the study. In addition, the protocol has been reviewed and funded by a scientific third party funding (grants from Nationales Centrum fuer Tumorerkrankungen (NCT)/Tumorzentrum Heidelberg and Kompetenzverbund Strahlenforschung (KVSF, 03NUK004A,C) of Bundesministerien fuer Bildung, Forschung und Umwelt (BMBF/BMU)). Patients are informed about the strict confidentiality of their personal data within this trial, but their pseudonymised medical records may be reviewed for trial purposes by authorized individuals other than their treating physician.

It has to be pointed out that the participation is voluntary and that the patient is allowed to refuse further participation in the protocol to any time point within the study. This trial is carried out in accordance to the current Declaration of Helsinki (sixth revision, 2008), the principles of "Good Clinical Practice" (GCP), and the Federal Data Protection Act. It is registered at the Clinical-Trials.gov protocol registration system (http://www.clinicaltrials.gov) and the assigned identification number is NCT01191632.

\section{Objective of the study}

The primary objective of this trial is the determination of the single fraction radiation dose, measured by the number of tumor infiltrating T-cells in the tumor. Secondary molecular objectives are the $\mathrm{T}$ cell activity in the resected tumor specimen, the density of regulatory $\mathrm{T}$ cells, and the frequency of tumor reactive $\mathrm{T}$ cells in blood and bone marrow in correlation with changes in the level of proteins involved in immune response or angiogenesis and transcriptomics in whole blood cells. Further clinical secondary objectives are acute radiogenic toxicity, postoperative morbidity and mortality, local control, recurrence patterns, overall survival and quality of life. All clinical secondary objectives are presented in Table 2.

Currently, 35 patients are randomized. The duration of the trial is expected to last four more months.

\section{Table 2 Secondary endpoints}

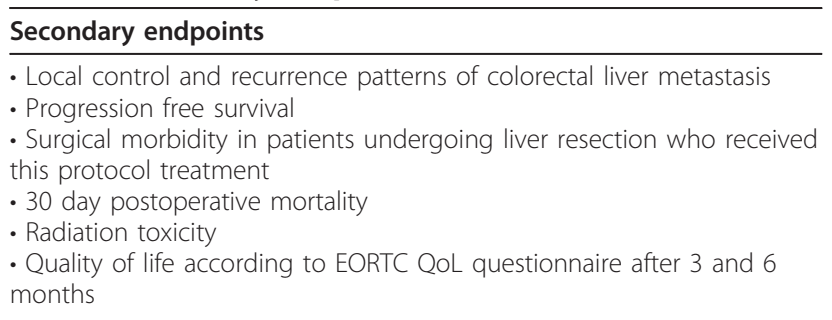

Usually, two patients per month can be enrolled into this study. Evaluation and reporting of the clinical and laboratory results will be done within three months after the end of recruitment and closing of the database.

\section{Statistical analysis}

This randomized controlled trial is planned as a comparison of four parallel groups:

Group 1: Dose = 0 Gy (control); Group 2: Dose $=0.5$ Gy; Group 3: Dose $=2$ Gy and Group 4: Dose $=5$ Gy.

Aim of the study is to determine the maximum active radiation dose with three different doses. This will be carried out hierarchically in two consecutive steps.

1. Step: Comparison of the three doses against the control group ("Many-to-one" comparison after Dunnet)

2. Step: Comparison of the three doses among each other ("all-subset" comparison)

Each of the two steps happens on multiple levels of significance with a p-value less than 0.05 . The power of each step should be at least $80 \%$.

Based on achieved information of a study by Galon et al. [20] in CRC patients, the density of tumor infiltrating $\mathrm{T}$ cells is a relevant prognostic parameter. Patients with a good prognosis had a mean of $\mathrm{x}$-good $=600 \mathrm{CD} 8 \mathrm{~T}$ cells $/ \mathrm{mm}^{2}$, the group with a bad prognosis had a mean of $\mathrm{x}$-bad $=370 \mathrm{CD} 8 \mathrm{~T}$ cells $/ \mathrm{mm}^{2}$. The standard deviation in both groups was $50 / \mathrm{mm}^{2}$. Further analysis revealed that patients with $\mathrm{x}=300$ tumor infiltrating CD8 T-cells $/ \mathrm{mm}^{2}$ in tumor tissue had a significantly better prognosis. Given this background, a clinical relevant difference between 100 and $200 \mathrm{CD} 8 \mathrm{~T}$-cells $/ \mathrm{mm}^{2}$ seems plausible. The number of cases should be sufficient for a difference of 150 CD8 T-cells $/ \mathrm{mm}^{2}$.

For the first step, 32 analyzable patients will be needed which equals eight patients for each of the four groups. The nominal power of ANOVA is around 90\%. For the second step (comparison of the best group, Hsu 2006), the power for $n=32$ is $95 \%$. Not all of the randomized patients could be analyzed with respect to the primary objective. A failure of $10-15 \%$ has to be assumed due to poor tissue quality or technical problems. Therefore, 40 patients should be randomized.

The calculated number of cases was carried out with PASS 2005 (Hintze J, 2004): NCSS and PASS. Number Cruncher Statistical Systems. Kaysville, Utah. http:// www.ncss.com) with the procedure for multiple comparisons by Jason C. Hsu (1996) (Multiple Comparisons: Theory and methods, Chapman \& Hall). The analysis of the primary objective results confirmatory on a multiple level of significance of $p<0.05$ by ANOVA based on the calculated numbers of cases. The analysis of the secondary objective results is descriptive by determining the means, the quartiles as location parameter and the standard deviation and range as dispersion parameters. 
The correlation between the parameters will be quantified by the Pearson or Spearman test. The EORTC QLY-C30 single-item and multi-item subscales will be scored and linearly transformed according to algorithms recommended by the EORTC. Using two-sample t-tests for continuous and Pearsons's $\mathrm{X}^{2}$ Test for categorical variables, the intervention and the control arms will be compared in terms of clinical characteristics at time of randomisation and in terms of baseline QOL scores. Repeated measures ANOVA will be applied to test for changes in the continuous secondary endpoints - e.g. plasma cytokine levels- as a function of treatment arms.

\section{Follow up}

A routine follow up for cancer patients will be done including physical examination of the patients, tumor markers (CEA), CT/MRI, chest X-ray every three months. Disease-free survival and overall survival will be recorded. Notably, the further clinical management and postoperative treatment of the patients will not be influenced by the results of this study. The decision whether patients will receive an adjuvant treatment (e.g. chemotherapy) is left to the discretion of the treating oncologist.

\section{Data management and quality assurance}

The coordination of this trial lies by the Department of Surgery and the Radiation Oncology Department of the German Cancer Research Center (DKFZ). Data regarding $\mathrm{T}$ cell detection in blood and bone marrow of all patients will be entered in a password-protected database at the Department of General-, Visceral- and Transplantation Surgery, University of Heidelberg and at the German Cancer Research Center. The clinical data of all included patients will be centrally collected in a database located at the Department of Radiation Oncology at dkfz. The clinical and laboratory data will be merged in the password-protected database at the Department of General-, Visceral- and Transplantation Surgery, University of Heidelberg, Germany and at the DKFZ.

\section{Discussion}

The primary objective of this clinical phase I/II study is to investigate if tumor conformal low dose external beam radiation using $6 \mathrm{MV}$ photons will lead to an increased number of tumor infiltrating $\mathrm{T}$ cells at two days after irradiation in resectable liver metastases of colorectal cancer.

The efficient radiotherapeutic treatment of colorectal liver metastasis is still a challenging task. Eradicating radiation doses are only applicable for small colorectal liver metastases because of the high radiation toxicity to the liver [21]. Therefore, the development of new therapeutic strategies is required. Cellular immunotherapy is a promising complimentary tumor treatment principle. Although the ability of tumor rejection of tumor specific $\mathrm{T}$ lymphocytes is well documented $[22,23]$, the effectiveness of this approach could not be translated into clinical trials. It appears to be essential, that the tumor context has to be specifically modulated to raise the efficiency for immunotherapy.

Low dose ionizing radiation offers the possibility of a selective induction of a proinflammatory milieu. In contrast to systemic adjuvants, a localized synergistic tumor effect is possible by causing a release of tumor antigens from necrotic tumor cells and depletion of regulatory $\mathrm{T}$ cells [24]. Ionizing radiation was initially considered as an immune-suppressive treatment modality [25], but it has also revealed its potential to enhance immunity.

Therefore, the hypothesis for the current study was that an improved $\mathrm{T}$ cell infiltration and activation in the tumor as well as a superior systemic $\mathrm{T}$ cell immunity against tumor antigens can be induced by low dose radiation therapy. However, the role of radiation as an independent immune-enhancer is still under investigation. While encouraging preclinical data are now available, their translation to the clinic - alone or in combination with other forms of immune therapy - is just beginning.

\section{Acknowledgements}

This work is supported in part by grants from Nationales Centrum fuer Tumorerkrankungen (NCT)/Tumorzentrum Heidelberg and Kompetenzverbund Strahlenforschung (KVSF, 03NUK004A, C) of Bundesministerien fuer Bildung, Forschung und Umwelt (BMBF/BMU).

\section{Author details}

${ }^{1}$ Department of General, Visceral and Transplantation Surgery, University Hospital Heidelberg, Germany. ${ }^{2}$ Department of Radiation Oncology, German Cancer Research Center and University Hospital Center, Heidelberg, Germany. ${ }^{3}$ Translational Immunology Unit, German Cancer Research Center, Heidelberg, Germany.

\section{Authors' contributions}

CR participated in the study design, trial coordination and conduction, patient recruitment, translational studies, and wrote the manuscript. CT participated in protocol design, ethical committee and BfS applications, patient recruitment and translational studies. HSW participated in developing the protocol concept, in the ethical committee application, trial coordination and patient recruitment. NNR participated in the patient recruitment and trial coordination. MK participated in the patient recruitment and trial coordination. FK carried out the immuno monitoring and participated in trial coordination. FR participates in trial conduction.

JD participates in trial conduction. LE made the statistics. MWB participates in trial conduction. PB participated in developing the study concept, trial conduction and coordinated the immuno monitoring. PEH coordinated the study concept, the trial conduction and translational studies and protocol design, led the approval committee applications. JW participated in developing the study concept, and trial coordination and conduction. All authors read and approved the final manuscript.

\section{Competing interests}

The authors declare that they have no competing interests. 
Received: 8 February 2011 Accepted: 30 September 2011

Published: 30 September 2011

\section{References}

1. Mensink PB, Kolkman JJ, Van Baarlen J, Kleibeuker JH: Change in anatomic distribution and incidence of colorectal carcinoma over a period of 15 years: clinical considerations. Dis Colon Rectum 2002, 45:1393-1396.

2. Leonard GD, Brenner B, Kemeny NE: Neoadjuvant chemotherapy before liver resection for patients with unresectable liver metastases from colorectal carcinoma. J Clin Oncol 2005, 23:2038-2048.

3. Reissfelder C, Rahbari NN, Koch M, Ulrich A, Pfeilschifter I, Waltert A, Muller SA, Schemmer P, Buchler MW, Weitz J: Validation of prognostic scoring systems for patients undergoing resection of colorectal cancer liver metastases. Ann SurgOncol 2009, 16:3279-3288.

4. Koch $M$, Beckhove $P$, Op dW, Autenrieth D, Wagner P, Nummer D, Specht S, Antolovic D, Galindo L, Schmitz-Winnenthal FH, et al: Tumor infiltrating $T$ lymphocytes in colorectal cancer: Tumor-selective activation and cytotoxic activity in situ. Ann Surg 2006, 244:986-992.

5. Bonertz A, Weitz J, Pietsch DH, Rahbari NN, Schlude C, Ge Y, Juenger S, Vlodavsky I, Khazaie K, Jaeger D, et al: Antigen-specific Tregs control T cell responses against a limited repertoire of tumour antigens in patients with colorectal carcinoma. Journal of Clinical Investigation 2009, 119:3311-3321.

6. Schmitz-Winnenthal FH, Volk C, Z'graggen K, Galindo L, Nummer D, Ziouta Y, Bucur M, Weitz J, Schirrmacher V, Buchler MW, et al: High frequencies of functional tumor-reactive $T$ cells in bone marrow and blood of pancreatic cancer patients. Cancer Res 2005, 65:10079-10087.

7. Nummer D, Suri-Payer E, Schmitz-Winnenthal FH, Bonertz A, Galindo L, Antolovic D, Koch M, Buechler MW, Weitz J, Schirrmacher V, et al: Role of tumor endothelium in CD4+ CD25+ regulatory $T$ cell infiltration of human pancreatic carcinoma. J Natl Cancer Inst 2007, 99:1188-1199.

8. Korangy F, Hochst B, Manns MP, Greten TF: Immunotherapy of hepatocellular carcinoma. Expert Rev GastroenterolHepatol 2010, 4:345-353.

9. Mougiakakos D, Choudhury A, Lladser A, Kiessling R, Johansson CC: Regulatory T cells in cancer. Adv Cancer Res 2010, 107:57-117, 57-117.

10. Ganss R, Ryschich E, Klar E, Arnold B, Hammerling GJ: Combination of Tcell therapy and trigger of inflammation induces remodeling of the vasculature and tumor eradication. Cancer Res 2002, 62:1462-1470.

11. North RJ: Gamma-irradiation facilitates the expression of adoptive immunity against established tumors by eliminating suppressor T cells. Cancer Immunollmmunother 1984, 16:175-181.

12. Szostak MJ, Kyprianou N: Radiation-induced apoptosis: predictive and therapeutic significance in radiotherapy of prostate cancer (review). Oncol Rep 2000, 7:699-706.

13. Demaria S, Bhardwaj N, McBride WH, Formenti SC: Combining radiotherapy and immunotherapy: a revived partnership. Int J Radiat Oncol Biol Phys 2005, 63:655-666.

14. Ciernik IF, Romero P, Berzofsky JA, Carbone DP: lonizing radiation enhances immunogenicity of cells expressing a tumor-specific T-cell epitope. Int J Radiat Oncol Biol Phys 1999, 45:735-741.

15. Abdel-Wahab Z, Dar MM, Hester D, Vervaert C, Gangavalli R, Barber J, Darrow TL, Seigler HF: Effect of irradiation on cytokine production, MHC antigen expression, and vaccine potential of interleukin-2 and interferon-gamma gene-modified melanoma cells. Cell Immunol 1996, 171:246-254.

16. Pang YY: The Brisbane 2000 terminology of liver anatomy and resections. HPB 2000; 2:333-39. HPB (Oxford) 2002, 4:99-100.

17. Crane $\mathrm{CH}$, Varadhachary $\mathrm{G}$, Pisters PW, Evans DB, Wolff RA: Future chemoradiation strategies in pancreatic cancer. SeminOncol 2007, 34:335-346.

18. Suh WW, Blackstock AW, Herman J, Konski AA, Mohiuddin M, Poggi MM, Regine WF, Cosman BC, Saltz L, Johnstone PA: ACR Appropriateness Criteria on resectable rectal cancer: expert panel on radiation oncologyrectal/anal cancer. Int J Radiat Oncol Biol Phys 2008, 70:1427-1430.

19. Timke C, Schmitz-Winnenthal FH, Klug F, Roeder FF, Reissfelder C, Rochet N, Koch M, Tjaden C, Büchler MW, Debus J, et al: Randomized controlled phase I/II study to investigate immune stimulatory effects by low dose radiotherapy in primarily operable pancreatic cancer. BMC Cancer 2011, 11:134.

20. Galon J, Costes A, Sanchez-Cabo F, Kirilovsky A, Mlecnik B, Lagorce-Pages C, Tosolini M, Camus M, Berger A, Wind P, et al: Type, density, and location of immune cells within human colorectal tumors predict clinical outcome. Science 2006, 313:1960-1964.

21. Weitz J, Koch M, Debus V, Hohler T, Galle PR, Buchler MW: Colorectal cancer. Lancet 2005, 365:153-165.

22. Beckhove P, Feuerer M, Dolenc M, Schuetz F, Choi C, Sommerfeldt N, Schwendemann J, Ehlert K, Altevogt P, Bastert G, et al: Specifically activated memory $T$ cell subsets from cancer patients recognize and reject xenotransplanted autologous tumors. J Clin Invest 2004, 114:67-76.

23. Feuerer M, Beckhove P, Bai L, Solomayer EF, Bastert G, Diel IJ, Pedain C, Oberniedermayr $M$, Schirrmacher $V$, Umansky $V$ : Therapy of human tumors in NOD/SCID mice with patient-derived reactivated memory T cells from bone marrow. Nat Med 2001, 7:452-458.

24. Zhang B, Bowerman NA, Salama JK, Schmidt H, Spiotto MT, Schietinger A, Yu P, Fu YX, Weichselbaum RR, Rowley DA, et al: Induced sensitization of tumor stroma leads to eradication of established cancer by T cells. J Exp Med 2007, 204:49-55.

25. Trott KR, Kamprad F: Radiobiological mechanisms of anti-inflammatory radiotherapy. Radiother Oncol 1999, 51:197-203.

\section{Pre-publication history}

The pre-publication history for this paper can be accessed here: http://www.biomedcentral.com/1471-2407/11/419/prepub

doi:10.1186/1471-2407-11-419

Cite this article as: Reissfelder et al:: A randomized controlled trial to investigate the influence of low dose radiotherapy on immune stimulatory effects in liver metastases of colorectal cancer. BMC Cancer 2011 11:419.

\section{Submit your next manuscript to BioMed Central and take full advantage of:}

- Convenient online submission

- Thorough peer review

- No space constraints or color figure charges

- Immediate publication on acceptance

- Inclusion in PubMed, CAS, Scopus and Google Scholar

- Research which is freely available for redistribution

Submit your manuscript at www.biomedcentral.com/submit
C) Biomed Central 